\title{
CONVERGENCE AND APPROXIMATION RESULTS FOR MEASURABLE MULTIFUNCTIONS
}

\author{
R. LUCCHETTI, N. S. PAPAGEORGIOU AND F. PATRONE
}

\begin{abstract}
In this note we consider measurable multifunctions taking values in a separable Banach space. We show that if $F(\omega) \subseteq$ s-lim $F_{n}(\omega)$, then any Castaing representation of the $F(\cdot)$ can be obtained as the strong limit of Castaing representations of the $F_{n}$ 's. We also prove that any weakly measurable multifunction is the Kuratowski-Mosco limit of a sequence of countably simple multifunctions. Then we show that in reflexive Banach spaces this approximation property is equivalent to weak measurability. Finally we discuss the problem of measurability of the inferior and w-superior limits of a sequence of measurable multifunctions.
\end{abstract}

1. Introduction. The purpose of this note is to extend some of the results of Salinetti and Wets [7] to separable Banach spaces. So we will prove a theorem on the convergence of Castaing representations and two others on the approximability of measurable multifunctions by countably valued multifunctions. The set convergence that we consider in this work is the Kuratowski-Mosco convergence of sets introduced by Mosco [6]. When the Banach space is finite dimensional, this convergence specializes to the well-known Kuratowski convergence of sets, which was used in [7]. Let us remark that Toma [8] extended the results of [7] on the measurability of the inferior and superior limits, from $\mathbf{R}^{n}$-valued multifunctions to multifunctions with values in Souslin, $\sigma$-compact, metric spaces. However in the Kuratowski-Mosco convergence the limit superior is taken with respect to the weak topology, which is not globally metrizable (unless of course $X$ is finite dimensional). So the results of Toma do not in general answer the question of whether the weak limit superior of a sequence of Banach space valued measurable multifunctions is measurable. We were unable to answer this question and to our knowledge this remains an interesting open problem. Our feeling is that in general the graph of the weak limit superior may not even be analytic. However, in a special case we have an answer. Namely assume that the Banach space $X$ is separable and that the sequence of measurable multifunctions is uniformly bounded by a weakly compact set $K$. Then recalling that the weak topology on $K$ is metrizable and that the Borel $\sigma$-fields generated by the weak and strong topologies coincide, we can conclude that the weak limit superior is measurable.

Received by the editors December 23, 1985 and, in revised form, April 25, 1986.

1980 Mathematics Subject Classification (1985 Revision). Primary 60C45, 54C40, 28 A45.

Key words and phrases. Kuratowski-Mosco convergence, measurable multifunction, Castaing representation, Aumann's selection theorem, complete $\sigma$-field, countably simple multifunction, Trojanski's renorming theorem.

This work was done while the second author was a visiting professor of C.N.R. at the Mathematics Department of Pavia. Support was provided by C.N.R. and N.S.F. Grant DMS-8403135. 
An approximation result, similar to our Theorem 3.2, was also proved by Tsukada [9] but under more restrictive hypotheses than ours. Namely $X$ was reflexive and both $X$ and $X^{*}$ had Fréchet differentiable norms.

Finally let us remark that concerning measurable multifunctions we will be using the terminology and the results of Castaing and Valadier [2]. Also we remind the reader that since our Banach space $X$ will be assumed to be separable, the concepts of Bochner (strong), Pettis (weak), and Borel measurability of point valued functions all coincide.

Our results can have useful applications in several areas of mathematics involving measurable multifunctions. All three theorems of this work can be used in the study of set valued random variables, which is an area that developed extensively in the last ten years (see Hiai $[\mathbf{1 1}]$ and the references therein). Also they can be used in the study of differential inclusions $\dot{x}(t) \in F(t, x(t))$ in which the orientor field $F(\cdot, \cdot)$ satisfies Carathéodory type conditions (see Aubin and Cellina [12]). Such set valued equations model several kinds of systems, like control systems, mathematical economics systems, biology systems, etc. Furthermore our results can be applied in the equilibrium theory of large economies, where measurable multifunctions are the main tool (see Klein and Thompson [13] and Khan [14]). Finally Theorem 3.3 shows that the Kuratowski-Mosco convergence, which was introduced in connection with variational inequalities and then found several other important applications (see Attouch [1]), is intimately related to the measurability of multifunctions.

2. Notations and hypotheses. Given $X$ a Banach space and $\left\{A_{n}\right\}_{n \geq 1} \subseteq$ $2^{X} \backslash\{\varnothing\}$ we define (see Mosco [6] and Attouch [1] )

$$
\mathrm{s}-\varliminf_{n \rightarrow \infty} A_{n}=\left\{x \in X: x=\mathrm{s}-\lim x_{n}, x_{n} \in A_{n}, n \geq 1\right\}
$$

and

$$
\mathrm{w}-\varlimsup_{n \rightarrow \infty} A_{n}=\left\{x \in X: x=\mathrm{w}-\lim x_{k}, x_{k} \in A_{n_{k}}, k \geq 1\right\} .
$$

We will say that the $A_{n}$ 's converge to $A$ in the Kuratowski-Mosco sense, denoted by $A_{n} \stackrel{\mathrm{K}-\mathrm{M}}{\longrightarrow} A$ if s-lim $A_{n}=A=\mathrm{w}-\varlimsup$

Also by $d_{A}(\cdot)$ we will denote the distance function from the set $A$, i.e. for all $x \in X, d_{A}(x)=\inf _{a \in A}\|x-a\|$.

Throughout this work we will be using the following notation:

$$
P_{f(c)}(X)=\{A \subseteq X: \text { nonempty, closed, (convex) }\} .
$$

A measurable space $(\Omega, \Sigma)$ is said to be complete if $\Sigma=\hat{\Sigma}$, where $\hat{\Sigma}=\bigcap_{\mu} \Sigma \mu$ with $\mu$ a positive, bounded measure on $(\Omega, \Sigma$, ) and $\Sigma \mu$ the $\mu$-completion of $\Sigma$. If $(\Omega, \Sigma, \mu)$ is a complete measure space, then $\Sigma=\hat{\Sigma}$. $\hat{\Sigma}$ is known as the universal $\sigma$-field generated from $\Sigma$.

By $B(X)$ we will denote the Borel $\sigma$-field of the Banach space $X$ endowed with the strong topology; $\Sigma \times B(X)$ denotes the product $\sigma$-field.

A multifunction $F: \Omega \rightarrow 2^{X} \backslash\{\varnothing\}$ (in particular a function) is said to be countably simple if $\Omega$ can be partitioned into a countable family of measurable subsets $\left\{\Omega_{1}, \Omega_{2}, \ldots, \Omega_{n}, \ldots\right\}$ s.t. $F(\cdot)$ is constant on each of the $\Omega_{i}$ 's (i.e. $F(\omega)=K_{i}$ for all $\omega \in \Omega_{1}$ and $K_{i}$ is a singleton if $F(\cdot)$ is point valued). 
3. The main results. First we have a result about the convergence of Castaing representations. Recall that if $F: \Omega \rightarrow 2^{X} \backslash\{\varnothing\}$ is a multifunction, then we say that $\left\{f_{n}(\cdot)\right\}_{n \geq 1}$ is a "Castaing representation" of $F(\cdot)$ if for every $n \geq 1, f_{n}(\cdot)$ is a measurable selector of $F(\cdot)$ and for all $\omega \in \Omega, F(\omega) \subseteq \operatorname{cl}\left\{f_{n}(\omega)\right\}_{n \geq 1}$. From Theorem 5.10 of Wagner [10], we know that if $(\Omega, \Sigma)$ is a measurable space with $\Sigma$ a Souslin family, $X$ is a Souslin space and $F: \Omega \rightarrow 2^{X} \backslash\{\varnothing\}$ is graph measurable, i.e. $\operatorname{Gr} F=\{(\omega, x) \in \Omega \times X: x \in F(\omega)\} \in \Sigma \times B(X)$, then $F(\cdot)$ admits a Castaing representation. If $\Sigma$ is complete then $\Sigma$ is a Souslin family.

THEOREM 3.1. Let $(\Omega, \Sigma)$ be a complete measurable space and $X$ a separable Banach space. Let $F_{n}, F: \Omega \rightarrow 2^{X} \backslash\{\varnothing\}$ be multifunctions that are graph measurable. Assume that for all $\omega \in \Omega, F(\omega) \subseteq \mathrm{s}-\underline{\lim } F_{n}(\omega)$ and let $\left\{v^{k}(\cdot)\right\}_{k \geq 1}$ be a Castaing representation of $F(\cdot)$. Then there exists a sequence $\left\{v_{n}^{k}(\cdot)\right\}_{k, n \geq 1}$ of Castaing representations of $F_{n}(\cdot)$ s.t. $v_{n}^{k}(\omega) \rightarrow v^{k}(\omega)$ for all $\omega \in \Omega$ and all $k \geq 1$.

PROOF. As we already mentioned Castaing representations do exist and so the theorem is not vacuously true.

Next we claim that the following is true:

"For any $v: \Omega \rightarrow X$, measurable selector of $F(\cdot)$, we can find measurable functions $z_{n}: \Omega \rightarrow X$, s.t. for every $\omega \in \Omega, z_{n}(\omega) \in F_{n}(\omega)$ and $z_{n}(\omega) \stackrel{\mathrm{s}}{\rightarrow} v(\omega)$."

To prove this claim we proceed as follows. For every $n \geq 1$ consider the multifunction $L_{n}: \Omega \rightarrow 2^{X}$ defined by $L_{n}(\omega)=\left\{x \in F_{n}(\omega):\|x-v(\omega)\| \leq\right.$ $\left.d_{F_{n}(\omega)}(v(\omega))+1 / n\right\}$. Clearly for every $n \geq 1$ and all $\omega \in \Omega, L_{n}(\omega) \neq \varnothing$. Also because $(\omega, y) \rightarrow d_{F_{n}(\omega)}(y)$ is a Carathéodory function, it is superpositionally measurable, and so $\omega \rightarrow d_{F_{n}(\omega)}(v(\omega))$ is measurable. Hence $(\omega, x) \rightarrow \varphi(\omega, x)=$ $\|x-v(\omega)\|-d_{F_{n}(\omega)}(v(\omega))$ is a Carathéodory function and so it is jointly measurable. Then note that

$$
\operatorname{Gr} L_{n}=\{(\omega, x) \in \Omega \times X: \varphi(\omega, x) \leq 1 / n\} \cap \operatorname{Gr} F_{n} \in \Sigma \times B(X) .
$$

Applying Aumann's selection theorem (see Castaing and Valadier [2, Theorem III22 , p. 74]) we get $z_{n}: \Omega \rightarrow X$ measurable s.t. $z_{n}(\omega) \in L_{n}(\omega)$ for all $\omega \in \Omega$. So $z_{n}(\omega) \in F_{n}(\omega)$ and $\left\|z_{n}(\omega)-v(\omega)\right\| \leq d_{F_{n}(\omega)}(v(\omega))+1 / n$ for all $n \geq 1$ and all $\omega \in \Omega$. Because $v(\omega) \in F(\omega)$ and by hypothesis $F(\omega) \subseteq \mathrm{s}-\underline{\lim } F_{n}(\omega)$, we have that $d_{F_{n}(\omega)}(v(\omega)) \rightarrow 0$ for all $\omega \in \Omega$. So $\left\|z_{n}(\omega)-v(\omega)\right\| \rightarrow 0$ and this proves the claim.

Now let $\left\{v^{k}(\cdot)\right\}_{k \geq 1}$ be a Castaing representation of $F(\cdot)$. Let $\left\{z_{n}^{k}(\cdot)\right\}_{k, n \geq 1}$ be measurable functions as in the claim. For every $n \geq 1$, take $\left\{w_{n}^{k}(\cdot)\right\}_{k \geq 1}$ to be a Castaing representation of $F_{n}(\cdot)$. Define

$$
v_{n}^{k}(\omega)= \begin{cases}z_{n}^{k}(\omega) & \text { if } k \leq n \\ w_{n}^{k-n}(\omega) & \text { if } k>n\end{cases}
$$

It is very easy to see that $\left\{v_{n}^{k}(\cdot)\right\}_{k \geq 1}$ is a Castaing representation of $F_{n}(\cdot)$ and that for all $k \geq 1$ and all $\omega \in \Omega, v_{n}^{k}(\omega) \rightarrow v^{k}(\omega)$ as $n \rightarrow \infty$. Q.E.D.

Now we will prove a useful approximation result for a large class of multifunctions. Namely we will show that every multifunction $F: \Omega \rightarrow P_{f c}(X)$ which is weakly measurable, i.e. for all $U \subseteq X$ nonempty and strongly open, $F^{-}(U)=\{\omega \in$ $\Omega: F(\omega) \cap U \neq \varnothing\} \in \Sigma$, can be approximated by countably simple multifunctions with closed (and if we want also convex) values. 
THEOREM 3.2. Let $(\Omega, \Sigma)$ be a measurable space and $X$ a separable Banach space. Let $F: \Omega \rightarrow P_{f c}(X)$ be a weakly measurable multifunction. Then:

A. There exist multifunctions $F_{n}: \Omega \rightarrow P_{f}(X)$ which are weakly measurable, countably simple, with values that are finite sets and $F_{n}(\omega) \stackrel{\mathrm{K}-\mathrm{M}}{\longrightarrow} F(\omega)$ for all $\omega \in \Omega$.

B. There exist multifunctions $G_{n}: \Omega \rightarrow P_{f c}(X)$ which are weakly measurable, countably simple and $G_{n}(\omega) \stackrel{\mathrm{K}-\mathrm{M}}{\longrightarrow} F(\omega)$ for all $\omega \in \Omega$.

PROOF. Let $\left\{f_{k}(\cdot)\right\}_{k \geq 1}$ be a Castaing representation for $F(\cdot)$ (its existence is guaranteed by Theorem III.8, p. 66 of Castaing and Valadier [2]). Define

$$
\hat{F}_{n}(\omega)=\left\{f_{1}(\omega) \cdots f_{n}(\omega)\right\}
$$

For every $n \geq 1$, we can find $s_{n}^{1}(\cdot), \ldots, s_{n}^{n}(\cdot)$ countably valued, measurable functions s.t. $\sup _{\omega \in \Omega}\left\|f_{i}(\omega)-s_{n}^{i}(\omega)\right\|<1 / n$ for all $i \in\{1, \ldots, n\}$ (see [2, p. 61]). Define $F_{n}(\omega)=\left\{s_{n}^{1}(\omega), \ldots, s_{n}^{n}(\omega)\right\}$. Clearly $F_{n}(\cdot)$ is weakly measurable countably simple and its values are finite sets. We claim that $F_{n}(\omega) \stackrel{\mathrm{K}-\mathrm{M}}{\longrightarrow} F(\omega)$ for all $\omega \in \Omega$. To this end, let us first note that $\hat{F}_{n}(\omega) \stackrel{\mathrm{K}-\mathrm{M}}{\longrightarrow} F(\omega)$ for all $\omega \in \Omega$. This is true because $\left\{\hat{F}_{n}(\cdot)\right\}_{n \geq 1}$ is an increasing sequence multifunction, so s- $\lim \hat{F}_{n}(\omega)=\overline{\bigcup_{n \geq 1} \hat{F}_{n}(\omega)}$. But note that $\bigcup_{n \geq 1} \hat{F}_{n}(\cdot)$ is a Castaing representation for $F(\cdot)$. So s- $\underline{\lim } \hat{F}_{n}(\omega)=$ $\overline{\bigcup_{n \geq 1} \hat{F}_{n}(\omega)}=F(\omega)$. On the other hand since $\hat{F}_{n}(\omega) \subseteq F(\omega)$ for all $n \geq 1$ and all $\omega \in \Omega$, we have that $\mathrm{w}-\varlimsup \overline{\lim } \hat{F}_{n}(\omega) \subseteq F(\omega)$. Hence finally we have that $\hat{F}_{n}(\omega) \stackrel{\text { K-M }}{\longrightarrow}$ $F(\omega)$ for all $\omega \in \Omega$.

Now let us prove that for all $\omega \in \Omega, F(\omega) \subseteq$ s- $\underline{\lim } F_{n}(\omega)$. So let $x \in F(\omega)$. Then we can find $y_{n} \in \hat{F}_{n}(\omega)$ s.t. $y_{n} \stackrel{\mathrm{s}}{\rightarrow} x$. From the definition of $\hat{F}_{n}(\cdot), y_{n}=f_{i_{n}}(\omega)$, $i_{n} \in\{1, \ldots, n\}$ (we select one of the indices that satisfy this property). Defining $x_{n}=s_{n}^{i_{n}}(\omega)$ we have that $x_{n} \stackrel{\mathrm{s}}{\rightarrow} x$ and this shows that $F(\omega) \subseteq \mathrm{s}-\underline{\lim } F_{n}(\omega)$.

We also need to show that for all $\omega \in \Omega$, w- $\varlimsup \digamma_{n}(\omega) \subseteq F(\omega)$. To prove this let $x_{k} \in F_{n_{k}}(\omega)$ s.t. $x_{k} \stackrel{\mathrm{w}}{\rightarrow} x$. We have $x_{k}=s_{n_{k}}^{i_{k}}(\omega)$ with $i_{k} \in\left\{1, \ldots, n_{k}\right\}$ (again we select one of the indices satisfying this property). But recall that $\| f_{i_{k}}(\omega)-$ $s_{n_{k}}^{i_{k}}(\omega) \|<1 / n_{k}$. Hence $f_{i_{k}}(\omega) \stackrel{\mathrm{w}}{\longrightarrow} x$. Since $f_{i_{k}}(\omega) \in F(\omega)$ for all $k \geq 1$ and $F(\omega)$ is weakly closed (being convex), we get that $x \in F(\omega)$. This shows that $\mathrm{w}-\varlimsup F_{n}(\omega) \subseteq F(\omega)$. Therefore for all $\omega \in \Omega, F_{n}(\omega) \stackrel{\mathrm{K}-\mathrm{M}}{\longrightarrow} F(\omega)$ and this proves A.

To get B simply define $G_{n}(\omega)=\operatorname{conv} F_{n}(\omega)$. It is easy to see that $G_{n}(\cdot)$ is weakly measurable. So we only need to show that $\mathrm{w}-\overline{\lim } G_{n}(\omega) \subseteq F(\omega)$. To this end take $x_{k} \in G_{n_{k}}(\omega)$ s.t. $x_{k} \stackrel{\mathrm{w}}{\rightarrow} x$. Then $x_{k}=\sum_{j=1}^{n_{k}} \lambda_{j} z_{j}, z_{j} \in F_{n_{k}}(\omega)$. We have

$$
x_{k}=\sum_{j=1}^{n_{k}} \lambda_{j}\left(z_{j}-f_{j}(\omega)\right)+\sum_{j=1}^{n_{k}} \lambda_{j} f_{j}(\omega)=u_{k}+w_{k}
$$

where $f_{j}(\omega) \in \hat{F}_{n_{k}}(\omega)$ and $\left\|z_{j}-f_{j}(\omega)\right\|<1 / n_{k}$ for all $j \in\left\{1, \ldots, n_{k}\right\}$. So $u_{k} \stackrel{\mathrm{s}}{\rightarrow} 0$. Hence $w_{k}=s_{k}-u_{k} \stackrel{\mathrm{w}}{\rightarrow} x$. But $w_{k} \in F(\omega)$. So $x \in F(\omega)$, which proves that w- $\varlimsup G_{n}(\omega) \subseteq F(\omega)$. That $F(\omega) \subseteq$ s- $\underline{\lim } G_{n}(\omega)$ follows from the fact that $F(\omega) \subseteq$ s- $\underline{\lim } F_{n}(\omega)$. Q.E.D. 
Assume that $X$ is a separable, reflexive Banach space. Then we have an equivalence between the approximability of $F(\cdot)$ by countably simple multifunctions and weak measurability. One implication was already proved in Theorem 3.2. So we need to show that the approximation property (which Tsukada [9] calls strong measurability) implies that $F(\cdot)$ is weakly measurable. But from Theorem 2.5 of [9] we know that $G_{n}(\omega) \stackrel{\mathrm{K}-\mathrm{M}}{\longrightarrow} F(\omega)$ implies that for all $x \in X, d_{G_{n}(\omega)}(x) \rightarrow d_{F(\omega)}(x)$ and so for all $x \in X, \omega \rightarrow d_{F(\omega)}(x)$ is measurable which, by Theorem III.9, p. 67 of [2], is equivalent to weak measurability. Furthermore Tsukada [9] proved that if $X$ is a separable Banach space s.t. both the space and its dual have Fréchet differentiable norms, then the Kuratowski-Mosco convergence of sets in $P_{f c}(X)$ is metrizable by a separable metric $\delta$. Then using that metric we can have a Borel $\sigma$-field $B_{\delta}\left(P_{f c}(X)\right)$ and speak about the $\left(\Sigma, B_{\delta}\left(P_{f c}(X)\right)\right)$-measurability of $F(\cdot)$. Tsukada [9, Theorem 4.2] proved that in a Banach space $X$ as above, this Borel measurability is equivalent to weak measurability. But if $X$ is a separable, reflexive Banach space, we know from Trojanski's theorem (see [3, Corollary 3, p. 167]) that $X$ can be equivalently renormed so that both the space and its dual have Fréchet differentiable norms. So the $\left(\Sigma, B_{\delta}\left(P_{f c}(X)\right)\right)$-measurability does not depend on the particular norm on $X$. Hence we can state the following theorem.

THEOREM 3.3. If $(\Omega, \Sigma)$ is a measurable space, $X$ is a separable, reflexive Banach space, and $F: \Omega \rightarrow F_{f c}(X)$ is a multifunction, then the following statements are equivalent.

1. There exist weakly measurable, countably simple multifunctions $G_{n}: \Omega \rightarrow$ $P_{f c}(X)$ s.t. for all $\omega \in \Omega, G_{n}(\omega) \stackrel{\mathrm{K}-\mathrm{M}}{\longrightarrow} F(\omega)$.

2. $F(\cdot)$ is weakly measurable.

3. For all $x \in X, \omega \rightarrow d_{F(\omega)}(x)$ is measurable.

4. $F(\cdot)$ is $\left(\Sigma, B_{\delta}\left(P_{f c}(X)\right)\right)$-measurable.

REMARKS. 1. As we already mentioned, Tsukada [9] calls property 1 in the above theorem, strong measurability. We did not adopt this terminology, because the name "strong measurability" is already reserved in the literature for multifunctions that are the Hausdorff limit of a sequence of simple, measurable multifunctions. Note that the metric topology induced on $P(X)$ by $\delta(\cdot, \cdot)$ is strictly weaker than the Hausdorff metric topology, unless $X$ is finite dimensional in which case on bounded closed, convex sets these topologies are equivalent (see Mosco [6]).

2. If $(\Omega, \Sigma)$ is complete and $F: \Omega \rightarrow P_{f}(X)$, then weak measurability, measurability of the distance function, existence of a Castaing representation, and graph measurability are all equivalent (see [2 and 10]). In this case we simply call the multifunction measurable.

3. As we already mentioned in the introduction the question about the measurability of $\omega \rightarrow \mathrm{w}-\varlimsup \overline{\lim } F_{n}(\omega)$ remains in general unanswered. On the other hand the measurability of $\omega \rightarrow \mathrm{s}-\underline{\lim } F_{n}(\omega)$ presents no problems. In fact let $(\Omega, \Sigma)$ be a complete measurable space, $X$ a separable Banach space, and let $F_{n}: \Omega \rightarrow$ $2^{X} \backslash\{\varnothing\}$ be measurable multifunctions. From Kuratowski [5, p. 335] we know that s- $\underline{\lim } F_{n}(\omega)=\left\{x \in X: \lim d_{F_{n}(\omega)}(x)=0\right\}$. But from Himmelberg [4, Theorem 3.3, p. 56] we know that $\omega \rightarrow d_{F_{n}(\omega)}(x)$ is measurable. Hence $(\omega, x) \rightarrow d_{F_{n}(\omega)}(x)$ is a Carathéodory function and so it is jointly measurable. Thus $\lim d_{F_{n}(\omega)}(x)$ is 
jointly measurable and so $\operatorname{Gr}\left(\mathrm{s}-\underline{\lim } F_{n}(\cdot)\right)=\left\{(\omega, x) \in \Omega \times X: \lim d_{F_{n}(\omega)}(x)=0\right\} \in$ $\Sigma \times B(X) \Rightarrow \mathrm{s}-\underline{\lim } F_{n}(\cdot)$ is graph measurable and from Remark 2 we conclude that it is measurable.

ACKNOWLEDGMENT. The authors are most grateful to the referee for his scholarly comments and suggestions.

\section{REFERENCES}

1. H. Attouch, Variational convergence for functions and operators, Pitman, Boston, Mass., 1984.

2. C. Castaing and M. Valadier, Convex analysis and measurable multifunctions, Lecture Notes in Math., vol. 580, Springer, Berlin, 1977.

3. J. Diestel, Geometry of Banach spaces-Selected topics, Lecture Notes in Math., vol. 485, Springer, Berlin, 1975.

4. C. Himmelberg, Measurable relations, Fund. Math. 87 (1975), 53-72.

5. K, Kuratowski, Topology. I, Academic Press, New York, 1966.

6. U. Mosco, Convergence of convex sets and of solutions of variational inequalities, Adv. in Math. 3 (1969), 510-585.

7. G. Salinetti and R. Wets, On the convergence of closed valued measurable multifunctions, Trans. Amer. Math. Soc. 266 (1981), 275-289.

8. V. Toma, Quelques problèmes de mésurabilité de multifonctions, Séminaire d'Analyse Convexe, Montpellier 13, 1983, pp. 6.1-6.17.

9. M. Tsukada, Convergence of best approximations in smooth Banach spaces, J. Approx. Theory 40 (1984), 301-309.

10. D. Wagner, Survey of measurable selection theorems, SIAM J. Control. Optim. 15 (1977), 859-903.

11. F. Hiai, Convergence of conditional expectations and strong laws of large numbers for multivalued random variables, Trans. Amer. Math. Soc. 291 (1985), 613-627.

12. J. P. Aubin and A. Cellina, Differential inclusions, Springer, Berlin, 1984.

13. E. Klein and A. Thompson, Theory of correspondences, Wiley, New York, 1984.

14. M. A. Khan, On extensions of the Cournot-Nash theorem, Advances in Equilibrium Theory, (C. D. Aliprantis et al., eds.), Springer, New York, 1985.

Department of Mathematics, University of Milano, Milano, Italy

Department of Mathematics, University of Illinois, 1409 W. Green Street, URBANA, ILLINOIS 61801

Department of Mathematics, University of Pavia, Pavia, Italy 